\title{
Avaliação do estado trófico para o Rio Sapucaí-Guaçu
}

Evaluation of the Trophic State to Sapucai-Guaçu River

La evaluación del estado trófico de Sapucaí-Guaçu Río

Maria Patrícia Sales Castro

Doutoranda em Eng. Civil - Recursos Hídricos, UFC, Brasil. patricia.sales@gmail.com

Ticiana Fontoura Vidal

Doutoranda em Eng. Civil - Recursos Hídricos, UFC, Brasil. ticianafvidal@yahoo.com.br

Raimundo Oliveira de Souza

Professor Doutor, UFC, Brasil. rsouza@ufc.br 
O lançamento de matéria orgânica, substâncias tóxicas e nutrientes via efluentes domésticos e industrial, ou resultante do manejo inadequado do uso do solo, pode afetar a qualidade do ambiente para os organismos aquáticos ou mesmo a saúde humana, por meio da ingestão de águas contaminadas. Visando isso, o objetivo da presente pesquisa foi avaliar a variação temporal das características tróficas do rio Sapucaí-Guaçu, no estado de São

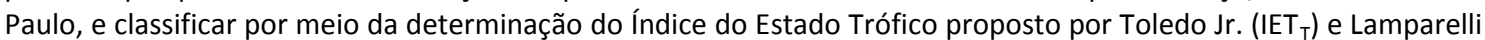
( $\left(E T_{L}\right.$ ) com os dados de qualidade de água entre os anos de 2012 a 2014. Os resultados apresentaram que os valores do Índice de Estado Trófico (IET), para os pontos avaliados no Rio Sapucaí-Guaçu, foram indicativos de estado de eutrofização, com tendência à hipereutrofização tanto para a metodologia utilizada por Toledo quanto à metodologia utilizada por Lamparelli. Foi possível observar também que as concentrações de fósforo encontradas nas amostragens foram superiores ao limite estabelecido na Resolução CONAMA n³57 de 2005, para os cursos de águas doces classe 2, provavelmente pelo fato do ponto do rio avaliado estar localizado em área urbana, e as maiores concentrações de fósforo nas águas estão associadas à carga de efluentes domésticos.

PALAVRAS-CHAVE: Eutrofização, Fósforo, Índice de Estado Trófico.

\section{ABSTRACT}

The release of organic matter, toxic substances and nutrients via domestic and industrial effluents, or resulting from inadequate management of land use can affect the quality of the environment to aquatic life or human health through the ingestion of contaminated water. Aiming at this, the objective of this study was to evaluate the temporal variation of trophic characteristics of Sapucai Guaçu River, in the state of São Paulo, and sort by determining the Trophic State Index proposed by Toledo Jr. (IET $T_{T}$ ) and Lamparelli (IET $T_{L}$ ) with the data quality of water between the years 2012 to 2014. The results showed that the values of the Trophic State Index (ETI) for the points evaluated in Sapucai Guaçu River, were indicative of eutrophication status, with tendency to hypertrophication both the methodology used by Toledo as the methodology used by Lamparelli. It was also observed that the phosphorus concentrations found in the samples were above the limit established in CONAMA Resolution No. 357 of 2005 to the fresh water Class 2, probably because the point of the appraised river is located in urban areas, and the higher phosphorus concentrations in water are associated with the load of domestic sewage.

KEYWORDS: Eutrophication, phosphorus, Trophic State Index.

\section{RESUMEN}

La liberación de la materia orgánica, nutrientes y sustancias tóxicas a través de los efluentes domésticos e industriales, o como resultado de una inadecuada gestión del uso del suelo puede afectar a la calidad del medio ambiente para la vida acuática y la salud humana a través de la ingestión de agua contaminada. Con el objetivo de esto, el objetivo de este estudio fue evaluar la variación temporal de las características tróficas de Sapucai río Guaçu, en el estado de Sao Paulo, y la especie mediante la determinación del índice de estado trófico propuesto por Toledo Jr. (IET ) y Lamparelli ( $\mathrm{IET}_{\mathrm{L}}$ ) con la calidad de los datos de agua entre los años 2012 al 2014. los resultados mostró que los valores del índice de estado trófico (ETI) de los puntos evaluados en Sapucai río Guaçu, eran indicativos del estado de eutrofización, con tendencia a hypertrophication tanto la metodología utilizada por Toledo como la metodología utilizada por Lamparelli. También se observó que las concentraciones de fósforo encontrados en las muestras estaban por encima del límite establecido en la Resolución CONAMA № 357 de 2005 para los cursos de agua dulce Clase 2, probablemente porque el punto del río de tasación se encuentra en las zonas urbanas, y las concentraciones de fósforo mayor en el agua están asociados con la carga de las aguas residuales domésticas.

PALABRA CLAVE: Eutrofización, el fósforo, el Índice de estado trófico. 


\section{INTRODUÇÃO}

Os impactos das atividades humanas nos ecossistemas aquáticos podem gerar uma série de problemas. Neste sentido, as pesquisas limnológicas têm despertado interesse crescente, visto que seus resultados podem ser amplamente aplicados para a conservação do ambiente aquático.

Segundo Tundisi (2005) a eutrofização é um fenômeno que afeta rios, lagos, reservatórios e tanques de abastecimentos, na superfície e águas costeiras. A eutrofização tem provocado a deterioração dos ecossistemas aquáticos e produzido impactos ecológicos, econômicos, sociais e na saúde pública.

Thomann e Mueller (1987) definem eutrofização como o crescimento excessivo de plantas aquáticas tanto planctônicas quanto aderidas, a níveis tais que sejam considerados como causadores de interferências com os usos desejáveis do corpo aquático.

A eutrofização é caracterizada pelo aumento na concentração de nutrientes, especialmente fósforo e nitrogênio, nos ecossistemas aquáticos, acarretando problemas como estímulo ao crescimento excessivo de algas, aumento da taxa de decomposição da matéria orgânica, com a redução do oxigênio dissolvido, e consequentemente, a deterioração da qualidade da água (ESTEVES, 1988; CAVENAGHI, 2003).

Segundo Mota (2006), a eutrofização pode levar à alteração no sabor, no odor, na turbidez e na cor da água; à redução do oxigênio dissolvido pode provocar a mortandade de peixe e outras espécies aquáticas, além da redução na balneabilidade da água.

Segundo Esteves (1988), o fosfato presente em ecossistemas aquáticos continentais tem origem em fontes naturais e artificiais. Dentre as fontes naturais, as rochas da bacia de drenagem constituem a fonte básica de fosfato. Outros fatores naturais que permitem o aporte de fosfato podem ser apontados, como o material particulado presente na atmosfera e o fosfato resultante da decomposição de organismos de origem alóctone.

Os Índices de qualidade da água foram propostos visando resumir as variáveis analisadas em um número, que possibilite observar a evolução da qualidade da água no tempo e no espaço e que sirva para facilitar a interpretação de variáveis ou indicadores.

Os nutrientes, principalmente, o fósforo e o nitrogênio, oriundos de fontes pontuais ou difusas encontram-se sob diversas formas no corpo aquático, mas nem todas são absorvidas pelo fitoplâncton.

Carlson (1977) desenvolveu um índice de estado trófico - IET baseado em equações que utilizam variáveis limnológicas, o qual passou a ser amplamente utilizado no monitoramento da qualidade da água em virtude da facilidade de aplicação e obtenção das variáveis de entrada no modelo.

O IET introduzido por Carlson (1977), modificado por Toledo Jr. et al. (1990), é baseado em informações relativas à biomassa fitoplanctônica presente em um determinado corpo de água, adotando-se as variáveis clorofila a, transparência (disco de Secchi) e fósforo total.

Em trabalho realizado por Quevedo (2009), no Rio Tietê, com índices de até vinte e um anos, foram encontradas concentrações entre 14 a mais de $4000 \mu \mathrm{g} \cdot \mathrm{L}^{-1}$ de fósforo total. A autora 
afirma que no alto Tietê e Tietê/Sorocaba a tendência de aumento gradual de fósforo ao longo dos anos é um fator que demonstra acompanhar o desenvolvimento econômico e populacional dessas bacias.

Em 1983, no Brasil, Toledo Jr. et al. realizaram estudos para adequar, a ambientes subtropicais, as equações desenvolvidas Carlson. Esta adaptação foi considerada necessária para reduzir distorções nos valores de classificação, uma vez que, por exemplo, as condições climáticas são diferentes daquelas das regiões de clima temperado.

Da mesma forma que Carlson, Toledo Jr. et al. (1990), aplicaram a análise de regressão linear aos valores de fósforo total, clorofila-a e transparência do disco de Secchi a reservatórios do estado de São Paulo. Como resultado de sua pesquisa foram produziadas as seguintes equações:

$\operatorname{IET}(P T)=10 x\left(6-\frac{\frac{\ln (80,32)}{P T}}{\ln (2)}\right)$

Lamparelli (2004) propôs índices (IET $\mathrm{L}_{\mathrm{L}}$ diferenciados para aplicação em ambientes lênticos (equação 2) e em ambientes lóticos (equação 3)

$\operatorname{IET}(P T)=10 x\left(6-\frac{0,42-0,36 x \ln (P T)}{\ln (2)}\right)-20$

Tanto para a equação 1 quanto a equação 2 temos que PT é a concentração de fósforo total em $\mu \mathrm{g} . \mathrm{L}^{-1}$.

Um índice de estado trófico funciona como um registro das atividades humanas nas várias bacias hidrográficas, além de oferecer subsídios para a formulação de planos de manejo e gestão de ecossistemas aquáticos, por meio de estratégias que visem a sustentabilidade dos recursos hídricos e que garantam os usos múltiplos da água, em médio e longo prazo.

Em síntese nesse trabalho teve-se o objetivo de avaliar a variação temporal das características tróficas do rio Sapucaí-Guaçu, no estado de São Paulo, por meio da determinação do Índice do Estado Trófico (IET), verificando a qualidade das águas entre os anos de 2012 a 2014.

\section{METODOLOGIA}

O presente trabalho foi realizado na microbacia hidrográfica do rio Sapucaí-Guaçu (Figura 1), o ponto de retirada da amostra foi na Ponte na Av. Emilio Lang Jr. com a Rua Engenheiro Prudente de Moraes, na saída para a estrada do Horto Florestal, localizado no município de Campos de Jordão (Figura 2). Cujas coordenadas geográficas são: 22 ㄴ' 58" S e 45 33' 36” W. O rio Sapucaí é um rio federal que banha os estados de São Paulo e Minas Gerais. Este nasce no município de Campos do Jordão, na serra da Mantiqueira. O rio percorre 248 km 
aproximadamente, até desaguar no reservatório da Usina Hidrelétrica de Furnas. O rio ao cruzar a zona urbana de Campos do Jordão e juntar-se a um de seus afluentes, o córrego das Perdizes, recebe o nome de rio Sapucaí-Guaçu. Somente cinco quilômetros antes da divisa com Minas Gerais o rio recebe o nome de Sapucaí.

Figura 1: Rede Hidrógrafica de Campos do Jordão

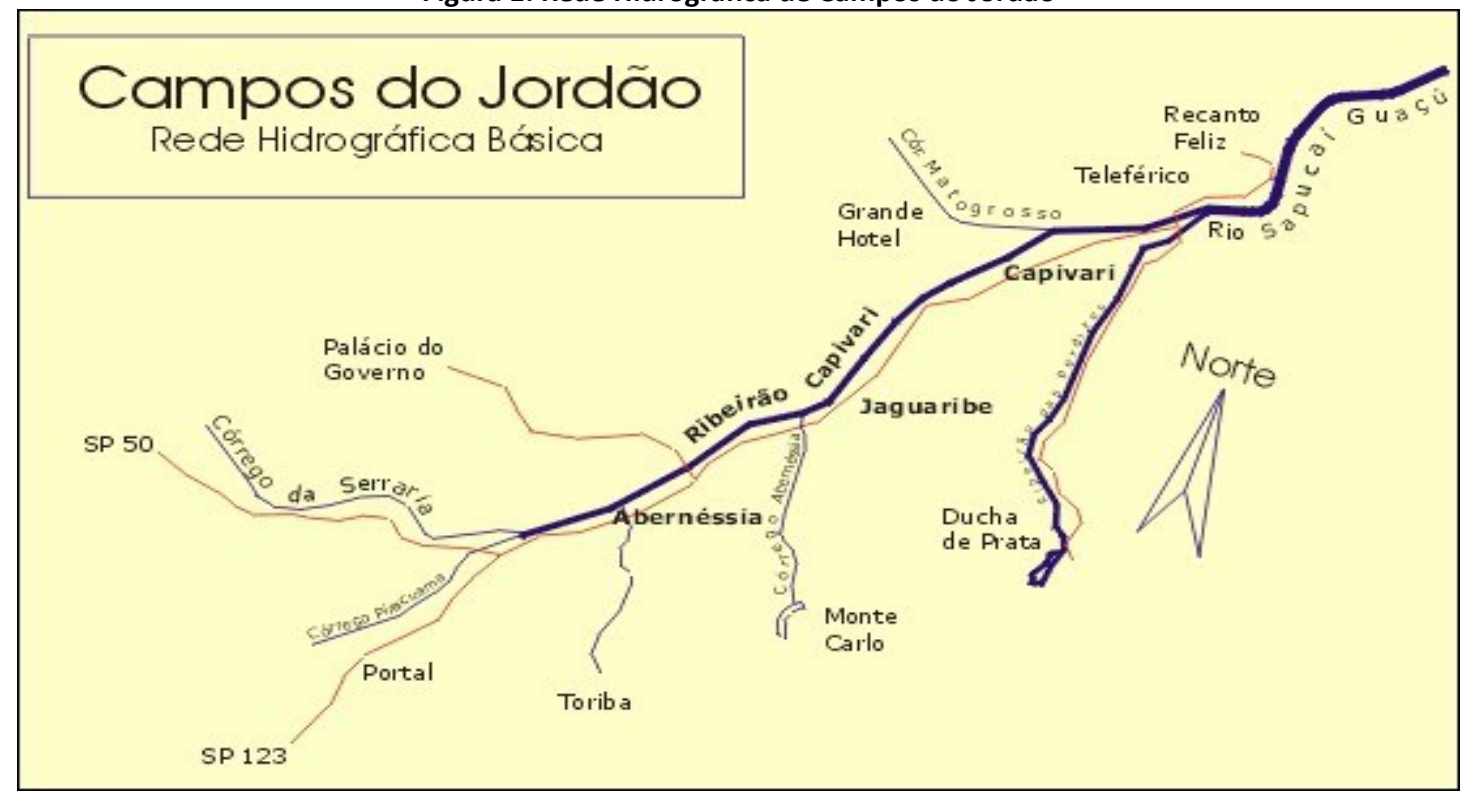

Fonte: Prefeitura de Campos de Jordão, 2016.

Figura 2: Ponto de coleta no Rio Sapucaí-Guaçu
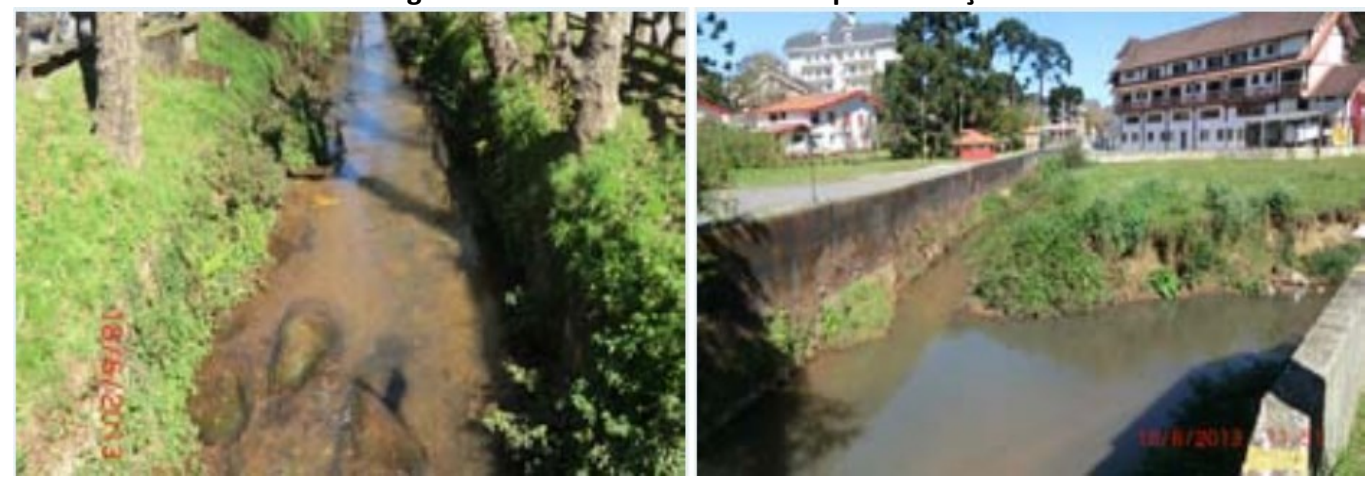

Fonte: CETESB, 2014.

Os dados de fósforo total e clorofila a foram retirados do site da CETESB, onde a CETESB anualmente publica um relatório anual sobre a qualidade das águas no estado de São Paulo. Este relatório é publicado em duas partes: Parte I - Águas Doces e Parte II - Águas Salinas e Salobras. Esta configuração tem como objetivo facilitar a consulta das informações relativas a essas duas condições ambientais. 
Duas metodologias relacionadas à variável fósforo foram empregadas para a classificação do nível de trofia no rio Sapucaí-Guaçu: a primeira refere-se à proposta por CARLSON (1977) adaptado por TOLEDO (1990) (Tabela 1) e a segunda refere-se à metodologia proposta por LAMPARELLI (2004) (Tabela 2)

A partir dos resultados obtidos, foram calculados os valores médios mensais de concentração de fósforo total $\left(P_{\text {total }}\right)$ e o índice de estado trófico (IET) modificado por Toledo (1990) e o IET proposto por Lamparelli (2004) e classificados de acordo com as tabelas 1 e 2, respectivamente. No caso do IET proposto por Lamparelli, fez-se uso das equações referentes a ambientes lóticos.

Tabela 1: Classificação do estado trófico segundo CARLSON (1977) e modificado por TOLEDO (1990).

\begin{tabular}{lcc}
\hline \multicolumn{1}{c}{ Estado trófico } & IET $_{\mathrm{T}}$ & $\begin{array}{c}\text { Fósforo total } \\
\left(\mu \mathrm{sL}^{-1}\right)\end{array}$ \\
\hline Hipereutrófico & $\mathrm{IET}=>74$ & $\mathrm{P}_{\mathrm{T}}>211$ \\
Eutrófico & $54<\mathrm{IET}<=74$ & $53<\mathrm{P}_{\mathrm{T}}<=211$ \\
Mesotrófico & $44<\mathrm{IET}<=54$ & $52<\mathrm{P}_{\mathrm{T}}<=27$ \\
Oligotrófico & $24<\mathrm{IET}<=44$ & $26<\mathrm{P}_{\mathrm{T}}<=7$ \\
Ultraoligotrófico & $\mathrm{IET}<=24$ & $\mathrm{P}_{\mathrm{T}}<=6$ \\
\hline
\end{tabular}

Fonte: TOLEDO, 1990

Tabela 2: Classificação do estado trófico segundo Lamparelli (2004).

\begin{tabular}{lcc}
\hline \multicolumn{1}{c}{ Estado trófico } & IET & $\begin{array}{c}\text { Fósforo total } \\
\left(\boldsymbol{\mu \mathrm { gL } ^ { - 1 } )}\right.\end{array}$ \\
\hline Hipereutrófico & $\mathrm{IET}=>67$ & $\mathrm{P}_{\mathrm{L}}>640$ \\
Supereutrófico & $63<\mathrm{IET}<=67$ & $296<\mathrm{P}_{\mathrm{L}}<=640$ \\
Eutrófico & $59<\mathrm{IET}<=63$ & $137<\mathrm{P}_{\mathrm{L}}<=296$ \\
Mesotrófico & $52<\mathrm{IET}<=59$ & $35<\mathrm{P}_{\mathrm{L}}<=137$ \\
Oligotrófico & $47<\mathrm{IET}<=52$ & $13<\mathrm{P}_{\mathrm{L}}<=35$ \\
Ultraoligotrófico & $\mathrm{IET}<=47$ & $\mathrm{P}_{\mathrm{L}}<=13$ \\
\hline
\end{tabular}

Fonte: LAMPARELLI, 2004 


\section{RESULTADOS}

Os valores de concentração do fósforo total e os índices de estado trófico para diferentes metodologias aplicadas no ponto de amostragem do Rio Sapucaí-Guaçu estão apresentados na Tabela 3.

Tabela 3: Resultados de Máximo, Mínimo, Média e Desvio-Padrão das concentrações de fósforo total $\left(P_{\mathrm{T}}\right)$ e seus respectivos índice de estado trófico (IET) obtidos para o Rio Sapucaí-Guaçu durante os anos 2012-2014.

\begin{tabular}{|c|c|c|c|}
\hline Data & $\begin{array}{l}\text { Fósforo total }\left(\mathrm{P}_{\mathrm{T}}\right) \\
\left(\mu \mathrm{g} \cdot \mathrm{L}^{-1}\right)\end{array}$ & $\mathrm{IET}_{\mathrm{T}}$ & $\mathrm{IET}_{\mathrm{L}}$ \\
\hline $18 / 02 / 20140$ & 110 & 83.10 & 84.91 \\
\hline 23/04/2014 & 170 & 84.71 & 84.73 \\
\hline $25 / 06 / 2014$ & 180 & 85.69 & 85.36 \\
\hline $27 / 08 / 2014$ & 310 & 90.14 & 87.23 \\
\hline $22 / 10 / 2014$ & 220 & 91.47 & 89.62 \\
\hline $10 / 12 / 2014$ & 210 & 93.27 & 91.35 \\
\hline $20 / 02 / 2013$ & 53 & 53.78 & 62.25 \\
\hline $24 / 04 / 2013$ & 152 & 61.38 & 64.99 \\
\hline $12 / 06 / 2013$ & 230 & 64.37 & 66.06 \\
\hline $21 / 08 / 2013$ & 220 & 64.05 & 65.95 \\
\hline $23 / 10 / 2013$ & 98 & 58.21 & 63.85 \\
\hline $11 / 12 / 2013$ & 315 & 66.64 & 66.88 \\
\hline 29/02/2012 & 7 & 39.18 & 57.00 \\
\hline $25 / 04 / 2012$ & 133 & 60.42 & 64.64 \\
\hline 27/06/2012 & 116 & 59.43 & 64.29 \\
\hline 22/08/2012 & 333 & 93.50 & 89.87 \\
\hline $24 / 10 / 2012$ & 106 & 82.97 & 84.94 \\
\hline $12 / 12 / 2012$ & 155 & 81.47 & 82.27 \\
\hline Média & 173.22 & 74.06 & 75.34 \\
\hline Máximo & 333 & 93.50 & 91.34 \\
\hline Mínimo & 7 & 39.17 & 56.99 \\
\hline Desvio-Padrão & 89.20 & 16.16 & 12.03 \\
\hline
\end{tabular}

As concentrações de fósforo nas amostras estão elevadas, o que caracteriza que esse curso de água como hipereutróficos. Segundo a Resolução CONAMA n³57 de 2005, para que um curso de água esteja na condição de classe 2 são necessários, entre outras avaliações, que as

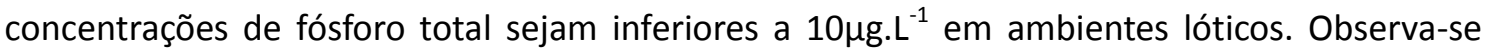
que, exceto a amostra coletada no dia 20/02/2012, estão com valores acima do estabelecido 
pela referida resolução. Destacando o dia 22/08/2012 onde teve a maior concentração de fósforo encontrada, isto se deve pelo fato do rio estar contido na zona urbana de Campos de Jordão e receber parte da poluição da cidade.

Nas Figuras 3 e 4 estão representados, respectivamente, os Índices de Estado Trófico modificado por Toledo (IET $T_{T}$ e o de Lamparelli (IET $)$ para o rio Sapucaí-Guaçu.

Figura 3: Valores de IET $_{T}$ obtidos na avaliação no Rio Sapucaí-Guaçu

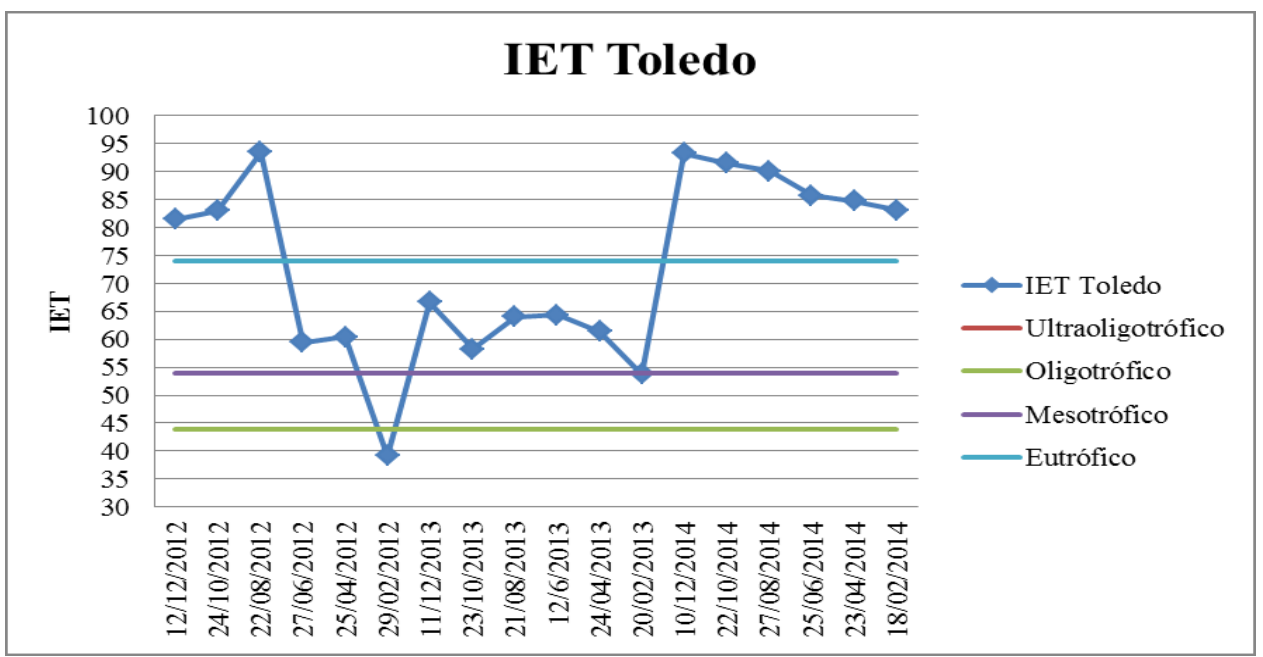

Fonte: Autor, 2016.

Figura 4: Valores de IETL obtidos na avaliação no Rio Sapucaí-Guaçu

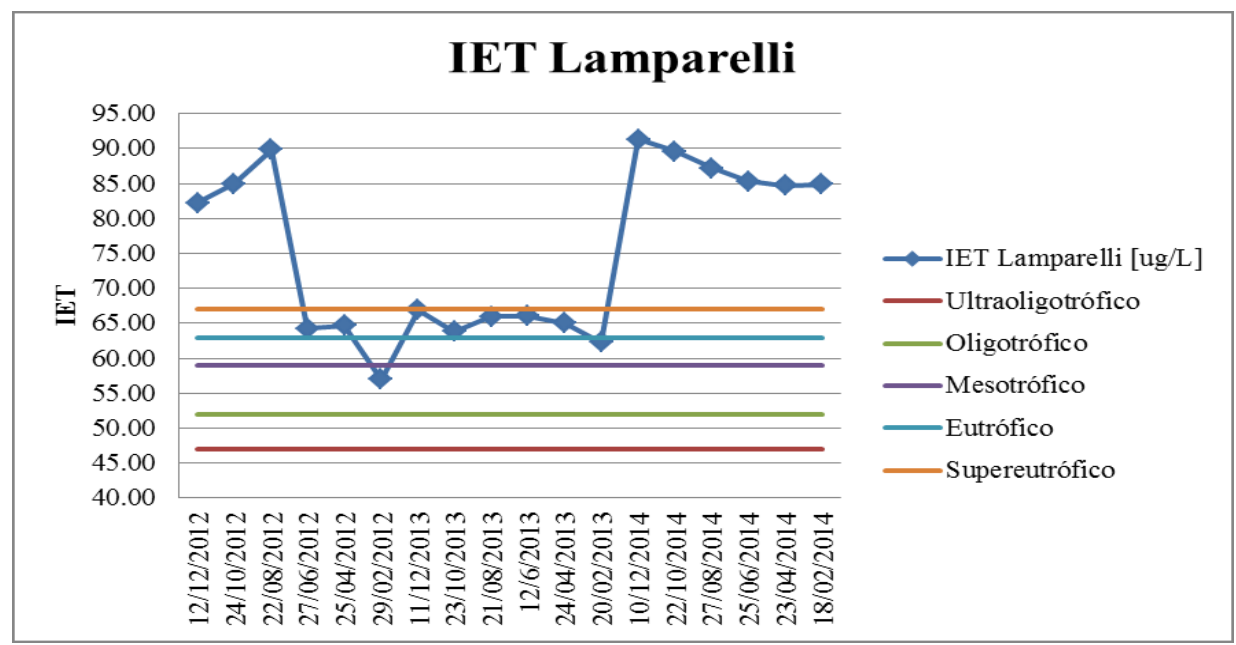

Fonte: Autor, 2016.

Tanto para o IET $\mathrm{IT}_{\mathrm{T}}$ quanto para o IET $\mathrm{I}_{\mathrm{L}}$ após verificar um período de pequenas variações entre os anos de 2012 e 2013, observa-se que ao longo do ano de 2014 os valores de IET tende a aumentar para ambos os casos. Constatou-se também a elevada concentração e consequentemente elevação do IET ao longo do ano de 2014, comparando com os anos 
anteriores. Onde no dia 10/12/2014 foram os valores de IET iguais a 93,27 e 91,35 respectivamente. Não foi possível verificar a influência das estações do ano em relação aos índices calculados.

Os resultados obtidos permitem dizer que o comportamento da variável avaliada é instável. Há períodos em que o estado trófico indica melhoria na qualidade da água e há períodos em que os valores alcançados tornam-se críticos, principalmente no ultimo ano de 2014 que apresentou tendência no aumento dos valores tanto para fósforo total e consequentemente IET.

Esse fato se deve as características do entorno dos cursos de águas. O Rio Sapucaí-Guaçu nasce na serra da Mantiqueira e ao cruzar a zona urbana de Campos do Jordão e juntar-se a um de seus afluentes, o córrego das Perdizes, recebe o nome de rio Sapucaí-Guaçu. Somente cinco quilômetros antes da divisa com Minas Gerais, este corpo ao longo de sua trajetória recebe os esgotos urbanos da cidade que carreiam material orgânico ricos em nutrientes e que supera a capacidade depurativa deste. Essas fontes pontuais de poluição conduzem a uma queda da qualidade das águas e a um intenso processo de eutrofização como observado no ambiente aquático avaliado.

\section{CONCLUSÕES}

Diante dos resultados obtidos, pode-se concluir que:

Os valores do Índice de Estado Trófico (IET), para os pontos avaliados no Rio Sapucaí-Guaçu, foram indicativos de estado de eutrofização, com tendência à hipereutrofização; As maiores concentrações de fósforo nas águas estão associadas à carga de efluentes domésticos, já que o ponto do rio avaliado está localizado em área urbana;

As concentrações de fósforo encontradas nas amostragens foram superiores ao limite estabelecido na Resolução CONAMA n³57 de 2005, para os cursos de águas doces classe 2; Não foi possível diferenciar tendências de variação nos IETs em função das estações do ano; A utilização do IET constitui metodologia de avaliação da qualidade de corpos de água bastante prática, facilitando a interpretação e divulgação dos resultados obtidos e, quando aplicado a corpos aquáticos de uma mesma região, permite a rápida avaliação comparativa do estado trófico dos mesmo;

O Índice de Estado Trófico modificado por Toledo Jr. mostrou-se mais conservador do que o Índice proposto por Lamparelli.

\section{AGRADECIMENTOS}

Os autores agradecem o apoio dado pelo CNPq e CAPES para a realização deste trabalho.

\section{REFERÊNCIAS BIBLIOGRÁFICAS}

BRASIL. Ministério do Meio Ambiente. Conselho Nacional de Meio Ambiente - CONAMA. Resolução n³57, de 17 de março de 2005: dispõe sobre a classificação dos corpos de água e diretrizes ambientais para o seu enquadramento, 
bem como estabelece as condições e padrões de lançamento de efluentes. Disponível em: http://www.mma.gov.br. Acesso em: 25 de abril de 2016.

CARLSON, R.E. A trophic state index for lakes. Limnology and Oceanography, v.22, n.2, p. 262-269, 1977

CAVENAGHI, A.L. Caracterização da qualidade de água e sedimento relacionados com a ocorrência de plantas aquáticas em cinco reservatórios da Bacia do rio Tietê. Botucatu: UNESP, 2003. 73f. (Tese de doutorado).

CETESB. Companhia Ambiental do Estado de São Paulo. Disponível em: http://aguasinteriores.cestesb.sp.gov.br/publicações-e-relatorios/. Acesso em 15 de março de 2016.

ESTEVES, F.A. Fundamentos de Limnologia. 2o ed. Rio de Janeiro: Interciência, 1988.

LAMPARELLI, M. C. Grau de trofia em corpos d'água do estado de São Paulo: avalição dos métodos de monitoramento. 238f. 2004. Tese (Doutorado em Ecologia Aplicada) - Universidade de São Paulo, São Paulo, 2004.

MOTA, S. Introdução à engenharia ambiental. 4. Ed. Rio de Janeiro: Abes, 2006. 388p.

THOMANN, R. VB., MUELLER, J. A. Principles of Surface Water Quality Modeling and Control. New York: Harper \& Row, 1987.

TOLEDO Jr., A. P. Informe preliminar sobre os estudos para obtenção de um índice para avaliação do estado trófico de reservatórios de regiões quentes tropicais. São Paulo: CETESB, 1990. 12p. (Relatório Interno CETESB).

TUNDISI, J. G. Água no século XXI: enfrentando a escassez. RiMa, iie. 2, Ed. São Carlos, 2005. 